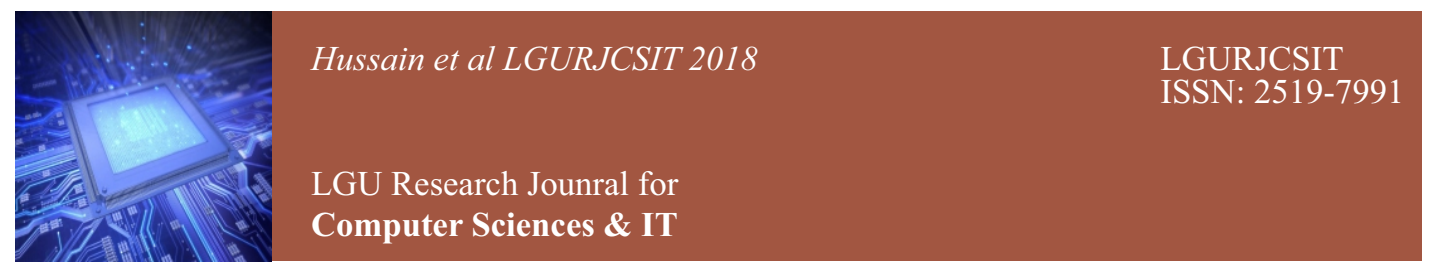

\title{
Ambient Intelligence
}

Muhammad Zunnurain Hussain ${ }^{1}, Z_{\text {Zaka Ullah }}^{2}$,Taimoor Hassan², Muhammad Zulkifl Hasan ${ }^{2}$

${ }^{1}$ Department of Computer Science, Bahria University Lahore Campus, ${ }^{2}$ Lahore Garrison University, Lahore, Pakistan, Zunnurain.bulc@bahria.edu.pk, zakaullah@lgu.edu.pk, taimoorhassan@lgu.edu.pk, zulkifl.hasan@lgu.edu.pk

\begin{abstract}
:
Ambient intelligence is electronic environment which are sensitive to the presence of people. It is the light of future technologies. All AMI subsystems are interact with each other to accomplish their goal. AMI applications which are directly interact to define the environment. It shows that human environment which are not produced this process. The AMI systems should identify the various emotions of human experience. The AMI systems can also highlight the communication of the user. The AMI systems which is consists of robots and sensors.
\end{abstract}

\section{Keyword:}

Ambient assisted living (AAL), Internet of Thing (IoT), Ubiquitous computing.

\section{INTRODUCTION}

Ambient intelligence is logical environment that supports the people in daily life. It has broad applications for commerce, medical and academic use. It is not used in the buildings or other things but it involved all of the environment which are backbone of the security environment. Ambient intelligence is a digital environment which are consists of some steps like sensing, acting, reasoning and interacting. Sensing are some of sensor networks which are connected to the door and floor for the security. it uses the ontological models and rule-3based reasoning for the purpose to facilitate the creation of an information systems.

\section{REVIEW OF LITERATURE}

World Health Organization has highlighted the population average age is increasing and no of elderly people is constantly growing. The model of ambient assisted living strives to develop modest intelligent systems that quality assist user in their life. The key feature of the system is 'user activities'. Smart homes systems generally utilize ambient sensors to measures physical quantities like temperature, lighting and humidity. AAL systems have sensor installed in smart phones, smart watches and wristbands which is used to check the behavior of the user. For every survey AAL tools improve the life of the people. Context information can be used by AAL system as input reasoning module, at the same time context attributes like location may be used to refine the interference process. AAL system that allow the user behavior and health conditions and other dangerous situations. The end goal of the system to improve the life quality and environmental conditions. The purpose of the multi-tier architecture for an AAL is that to understand the environment, the behavior of user and the description of context. The multitier architecture is composed of several modules like sensor manager which is responsible for data collecting from sensor. The activity module is the important part of architecture which describe the effectiveness of whole system. The environmental modeler which describe the effect of actions on environment. Reasoner module contains rule-based approach to 
interfere the user conditions. The experimental setting which describe the effectiveness of proposed AAL system in the application scenario of a smart phone. The first set of the organization gives the whole result of the system.

The internet of thing (IoT) is the most important model in the information and communication technology. It guides to all kinds of solutions which are used in the ambient intelligence. The IoT and AMI system has induced the interest of the IT students to their plan and applications. This system contains the language, protocol, devices and communications mechanism. All AMI subsystems are interact with each other to accomplish their goal. The technical AMI system is used to understand ICT areas like database design and applications or etc. AMI has been used for learning to understand the many concept of applications of the project. Three phases that identifies points from measuring and quality point of view. Tools and techniques support novice programmer for developing.

The reasoning which depend upon the skillful work and risk to human error. Reasoning rule shows several challenges for factor knowledge. In AMI the rule-based reasoning depend upon ontological models. The models which are main points of the AMI system for sharing knowledge of an environment. There are many ways, first we continue matching different on topologies. We discover source of difference and repair. After before maintain and repair them. Finally by seeing the inconsistency by various shapes of same on topologies.

Presently, AMI technology is used for research domain and implementation in SMEs. SMEs are required for precise methods, effective application of emerging skills to create computable assistance on short and medium term with the invention of large companies. The main aim of manufacturing SMEs to vigorously take part in this rebellion by applying emerging AMI technology. AMI based clarifications are combined with the ideas of ubiquitous computing and intelligent systems putting humans in the center technological development. By the research activities on specific AMI technologies there is no acknowledged description of AMI systems in manufacturing industry. The AMI systems in industry can be measured as control systems of computerization\& robotics processes. The orientation requires that AMI technologies offer theoretically optimization of the collaboration of human operators within their environment. The ambience is divided up in the manufacturing related ambience, covering procedure and plant etc. The intelligence of interaction of human operator with environment is managed by AMI systems as shown in this Figure.

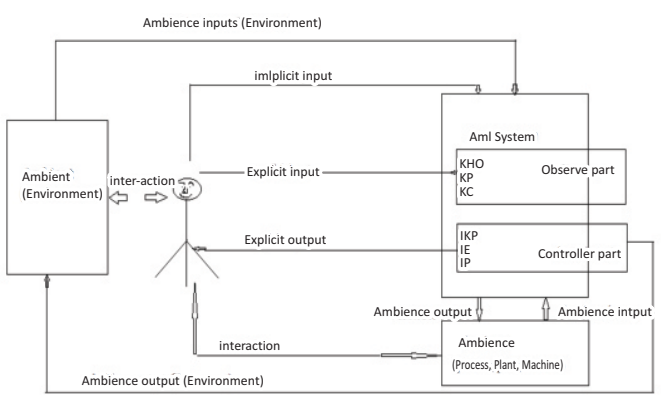

Figure 1: AMI System

The obvious efforts from $\mathrm{HO}$ to the AMI systems are the ordinary HO contributions like vocalized language, script, touchscreen and remark to the AMI system. The digital pen, trace pad, script acknowledgment and communicational acknowledgment are AMI technologies. The wireless handheld devices like PDASs, tablet PC or mobile phones. The biometric validation and sanction are AMI technologies. Videos consultation technology, mobile communication like Bluetooth etc. These are the examples for the relative among AMI structures and skills.

In ambient intelligence on condition that smart homes provide a sensor networks and intelligent systems, we are capable to automate the opinion and cautions. Curiosity is increasing from academics, companies and health authorities in the practice of computational intelligence practices to sustenance people for longer period in their homes and connected with friends. Ambient assisted living (AAL) endorses the creation of products, schemes and explanations for life support of people. The low cast dispensation power and wireless sensor shows that ambient computational intelligence has discovered for observing activities. The data we are used to the Activities of Daily Living for a number of people who are living in homes instrumented with sensors. The iCarer project which is used for monitoring daily living so that problem indicated. The sensor used were inert motion, opening doors sensors which are to detect which person enter the room. The whole 
system was exhibited in an ontology which are related to the sensor, rooms, actions and people.

Ambient intelligence research which are focused on the improvements of human interactions with smart applications. These improvements are the proposal of frameworks and platform that facilitate the development of dynamic applications. The internet of livings are to provide a global infrastructure for the information society. The present applications use devices that are vertically connected from the device to an external server that collects and processes the data. Applications and services are expected to ensure the privacy of the various data. The internet of things (IoT) based on intelligent agents. The classical works in ambient intelligence recommendboards that provide devices to build context-aware applications. This mechanism grip the data and events but some work show the disposition of these applications. As event is a software entity, the disposition of agents does not deal with high heterogeneity of hardware objects. The disposition tools contains solicitations and its infrastructure in which hardware entities of ambient systems are unified and used to support for running the applications. We use a graph theory to model hardware requirements, properties and their relations as shows an example of an applications description. The upper part shows various tasks and interfaces. The lower part shows arrangement pattern of applications and their requirements.

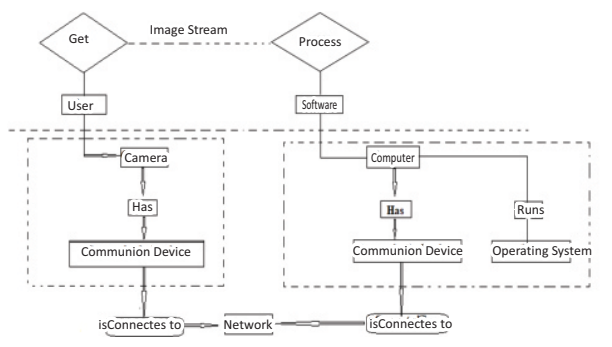

\section{Figure 2: Multi-agent system (MAS)}

In design and implementation we choose the Multi-agent system (MAS). This system facilitate local dispensation of data, the sovereignty of different parts of substructure and handle some aspects of privacy. In MAS the agents are able to the projection of applications w.r.t the available infrastructure.

AMI is the hallucination that technology will become imperceptible, embedded in our natural environs and content must be accessible to any user at any time and on any device. AMI becomes a certainty that should entirely envelop humans without coercing them. AMI changes the way we design embedded systems and have a great impression on the way we live. Application range from safe driving system, smart buildings, home security and human life. There is lot of works done for AMI. In AMI that offers the library of Networked Control Systems (NCS) blocks. NCS pronounce real process, a bob which is automated based on Mica-nodes. This nodes is used for AMI purposes. The real NCS serves as a exciting for proving AMI suitability of controllers.

In ambient intelligence all the evidence can be supported by user and intelligence can be provided by electronic devices as IoT. These devices can transfer with each other through data and information. The milieu of AMI is user friendly and favorable for applications like CAPT in one of popular application of AMI environment. CAPT is learning technology application that correct the articulation of user. CAPT also provide pointer by checking the fault pronunciation of user speech. After detecting the pronunciation CAPT also check the sentence and word. In AMI a smart-phone assisted pronunciation technique are also used in which articulation can be effective by lightweight word method. In AMI milieu user used this method to speak test and displayed on the smart-phone. After displaying the SAPT scrutinize the mistake.

AAL is the part of ambient intelligence. The system ambient aided living support safe life. The SSL is a task for current milieu situation. When we build a context formation in SSL we need multi-agent organization process. The method of the context formation by a number of researches which play an important part in AMI. Aim of the context to build high level thoughts of user actions. Ambient intelligence applied in every area like AAL because it based on the theoretical data source. This systems contain different language. These ambient intelligence are used in every aspect of life because it involved in sensors. All work is done by the sensor if a person enter the room then he feels the hot then fan is on. If he see T.V, then T.V is on. This is ambient intelligence.

Sensor networks are used to build AMI systems with applications for security, safety 
and living. Now a days, researches of service robots with wireless sensor network. Mobile robots maintain the data from wireless networks.

A major part which is implemented of AMI systems. The AMI systems which is consists of robots and sensors. The distributive ambient intelligence architecture (DAMIS) which provides a flexible infrastructure of robots and sensors. The multi-robots and sensors is based on robot operating system. The contribution of DAMIS is used robotic system with network of sensors over wireless, wires and layers. The DAMIS architecture is real world ambient intelligence. ROS is open source robotics software.

Computer education is introduced with the inventions of new technologies, devices and environment. The educational institutions provides the student skills and assign new topic like IoT and mobile computing. The internet of thing (IoT) based on network devices, protocol and applications. In any documentation and projects course some of the requirements are needed.

Table 1: Key features qualifying an AMI

\begin{tabular}{|l|l|}
\hline Features & Description \\
\hline Sensitive & Able to process sensor data. \\
Responsive & Able to response user need \\
Adaptive & Able to adopt to the context \\
Ubiquitous & Computing elements are everywhere \\
Intelligent & Artificial intelligence is enable for context awareness. \\
\hline
\end{tabular}

The AMI which uses size the skills of the students and teach about the new technologies. Some of the learning goals are achieved by the students. In this institutions all working in semester in which IoT and AMI definitions, requirements and design methodologies are studied. The practical programming systems like python, web protocol, and android development and collaboration tools are used. In the last exam the project of AMI systems are presented in the form of presentations. During exam two different aspects are subject to evaluation, the one is uploaded materials and second one is presentations. The final marks are is determined by adding two evaluations.

\section{Final AMI projects}

\begin{tabular}{|l|l|l|}
\hline Years & Projects & Descriptions \\
\hline 2014 & DoorOnPhone & Home intercom on smartphone \\
\hline 2015 & Track Down & Detect it something was stolen from bag \\
\hline 2015 & MarcoPoli & Sensor around the campus to identify critical areas \\
\hline
\end{tabular}

Some of new biometrics system are designed for security applications. These applications are traditional biometrics systems. Voice recognition applications contains two categories 1) speaker recognition and 2) speech recognition. Speech recognition is used for AMI environment and speaker recognition are used only for authentication process. It shows an AMI environment build on speech and speaker recognition. The speaker recognition consists of two categories one is text-dependent and second one is text-independent. The first requires user communications and second have not limitations. Text-dependent are greater than accurate than text-independent. Speaker recognition identify a person.

Simulation provides many performance with unlike framework to get knowledge for a particular environment. There are many simulators which are used specific purpose. For example social simulator simulate the human behaviors within human and environment. This is interesting approach for AMI simulator but deep simulation behavior is cyber-physical devices in AMI system. Network simulator can simulate detail behavior of networks but lack interaction between people and behavior in AMI system. Each of simulators can good explain to the AMI environment. By combining both simulators we can improve another simulator which contains simulator with both people by AMI systems. By the interaction of both simulators we obtain many benefits. The ambient technologies contains features like adaptive, sensitive, ubiquitous and intelligent.

Ambient intelligence is a new technology which are covered to the humans. Distributed embedded system for AMI which change the life. Applications vary from building which are composed all over the society where people lives. In this topic a simulation environment that provide network control system (NCS) blocks. These nodes is designed 
for AMI purposes. OLSR uses for messages to find their responses. This is protocol proactive routing protocol which build data actions by place of networks.

AMI is the type of machine intelligence. AMI applications which are split into independent systems. AMI can show the learner behaviors. In this topic we studied the new methodologies for AMI smart learning environment. By studying the smart teaching we some have some problems during working. Smart class uses many intelligence and applications. AMI class room promotes easy interactions. AMI is ability to make the student information access easier. It promote collaborate learning environment. AMI sensor cover the task of activity and provide service directly. Sensor of AMI environment is installed to observe the user activity. There are some sensor and their purposes given below.

Table 2: Sensors and purpose

\begin{tabular}{|l|l|}
\hline Type of sensors & Sensors purpose \\
\hline IR sensor & Lip movement \\
\hline Visual sensor & Book movement \\
\hline Magnetic sensor & Chair movement \\
\hline
\end{tabular}

\section{METHODOLOGY}

The techniques and hardware are involved in the progress of ambient intelligence systems are under of continuous evaluation. The use of protocols, wireless sensor networks and devices are involved to corporate new sources of information, these can be processed by the improvements of computing and reduced the cost of different devices. As a result there is a huge use in which new trends in wireless sensor networks and device can be applied. This issue will focus on the new trend in wireless sensor network and device which can also use in artificial intelligence, distributed intelligence for information processing. In this ambient intelligence all of the intelligence and system are involved like ambient assisted livings (AAL), multi-agents systems, context-aware computing, mobile computing and many other system are used in this ambient intelligence. I should more improve these systems to more update the network's sensor which can be used in the all over the ambient intelligence because this is basic need of the people to be facilitate these technologies and the basic usage of the ambient intelligence.

\section{FUTURE WORK}

In future the ambient intelligence is a new technology because low costs ambient technology is developed. This technology is used in all aspects of life like home, hospital, senior are center etc. it is rabidly field of information that has great impact in future.

\section{CONCLUSION}

The concept of ambient intelligence is new emerging technology. It is gaining importance due to its presence the subset of ambient intelligence. Ambient intelligence contributing many technologies such as reasoning, sensing, $\mathrm{HCI}$ and security. AMI should be easy to live in which we define the context awareness and natural awareness. In this work we conclude that the architecture for a complete AAL system which is used for the user activities and behavior or in the health conditions. This approach which is used to detect for AMI system which are new sensor. By using test bed QoL improved. The AMI definition and topologies that a part of AMI develop new technology. A smart-phoneassisted technique that provide AMI to user. The AMI Cource is used for the students that it obtains knowledge from the new technologies. This subject is introduce in 2014. AMI system is used for the identification of speech and speaker recognition method. The method to develop the user behavior which are obtained by sensors in AMI. It also shows the various forms of behavior data.

\section{References}

[1] Alessandra De Paola, An Ambient Intelligence System for Assisted Living, pp. 16.2017

[2] Fulvio Corno, Pain Points for Novice Programmers of Ambient Intelligence Systems: an Exploratory Study, pp. 250-255, 2017.

[3] Hamdi Aloulou, Detecting Inconsistencies in Rule-Based Reasoning for Ambient Intelligence, pp. 235-240, 2016. 
[4] Sebastian Scholze, "Ambient Intelligence Technologies for Industrial Working Environments in Manufacturing SMEs," pp. 1-8.2008.

[5] Caroline Langensiepen, "Uncertainty Measures in an Ambient Intelligence Environment,"pp. 1-5, 2017.

[6] Ferdinand Piette?†, "Intelligent Agents for Preserving Resource Privacy when Deploying Ambient Intelligence Applications," pp. 43-50, 2016.

[7] Ryoichiro Obakata, "performance evolutions of an ambient intelligence test bed for improving quality," pp. 484-487, 2016.

[8] I. JAESUNG LEE1, "SmartphoneAssisted Pronunciation Learning Technique for Ambient Intelligence," vol. 5, pp. 312-325, 2016.

[9] Peter Ivanov, "Forming the multi-modal situation context in ambient intelligence systems," pp. 7-12, 2015.
[10] Antonio Petitti, A Network of Stationary Sensors and Mobile Robots for Distributed Ambient Intelligence, pp. 28-34, 2016.

[11] Fulvio Corno, Training Engineers for the Ambient Intelligence Challenge, vol. 60, pp. 4049, 2016.

[12] Abhinav Anand?, Text-Independent Speaker Recognition for Ambient Intelligence Applications by Using Information Set Features, pp. 1-6, 2017.

[13] Álvaro Sánchez-Picot, Modeling and simulation of interactions among people and devices in Ambient Intelligence environments, pp. 734-739, 2016.

[14] Ryoichiro Obukata, Design of an Ambient Intelligence Testbed for Improving Quality of Life, pp. 714-719, 2016.

[15] S. R. Sujith Kanagarajan, Development of Ontologies for Modelling User Behaviour in Ambient Intelligence Environment, pp. 1-6, 2015. 